\title{
Uniplanar Log-Periodic Slot Antenna Fed by a CPW for UWB Applications
}

\author{
Shih-Yuan Chen, Member, IEEE, Po-Hsiang Wang, and Powen Hsu, Senior Member, IEEE
}

\begin{abstract}
A coplanar waveguide-fed uniplanar log-periodic slot antenna suitable for use in the ultra-wideband radio systems is presented. The in-band impedances and radiation performances are quite stable and satisfactory. A rejected narrow frequency band is further obtained within the wide bandwidth by inserting a pair of metallic stubs into two vertical slots of the proposed antenna. Experimental results reveal that the stub pair provides significant attenuation in the desired notched band but has little effect on the antenna operation outside of the notch. By means of a normalized antenna transfer function, the time-domain characteristics of the proposed antennas are also investigated. The transient responses show that the designs with and without the notched band are both acceptable for the pulsed systems.
\end{abstract}

Index Terms-Coplanar waveguides, log periodic antennas, slot antennas.

\section{INTRODUCTION}

$\mathbf{S}$ INCE the approval of ultra-wideband (UWB) by the Federal Communications Commission (FCC) in 2002 [1], the UWB technology has gained more and more popularity and become an important candidate for short-range high-speed indoor data communication applications [2], [3]. Nevertheless, the antenna design for UWB radio systems still poses several distinct challenges, including the relatively wideband operation and nearly invariable in-band performances. In addition, due to the low power operation of pulsed UWB systems, it is necessary to notch out portions of the 3.1 to $10.6 \mathrm{GHz}$ band to suppress the interference from existing wireless networking technologies, such as the IEEE 802.11a and the High Performance Radio Local Area Network (HIPERLAN/2), of which the spectrums are allocated within 5.15 to $5.825 \mathrm{GHz}$. Therefore, a UWB antenna possessing band-notched characteristics is more favorable in practical applications. However, to our knowledge, only a few works so far have focused on such category of UWB antennas [4]-[7]. In this paper, we propose a coplanar waveguide (CPW)-fed log-periodic slot antenna having a notched frequency band. The bandwidth and the center frequency of the notch are tunable, and a simple design formula is provided to facilitate allocating the notched band. Moreover, due to the compact size of the proposed antenna, the inherently dispersive properties of the log-periodic structure [8], [9] are reasonably suppressed.

Manuscript received December 16, 2005; revised February 10, 2006. This work was supported by the National Science Council, Taiwan, R.O.C., under Contract NSC 94-2752-E-002-002-PAE.

The authors are with the Department of Electrical Engineering and the Graduate Institute of Communication Engineering, National Taiwan University, Taipei 10617, Taiwan, R.O.C. (e-mail: phsu@cc.ee.ntu.edu.tw).

Digital Object Identifier 10.1109/LAWP.2006.873956

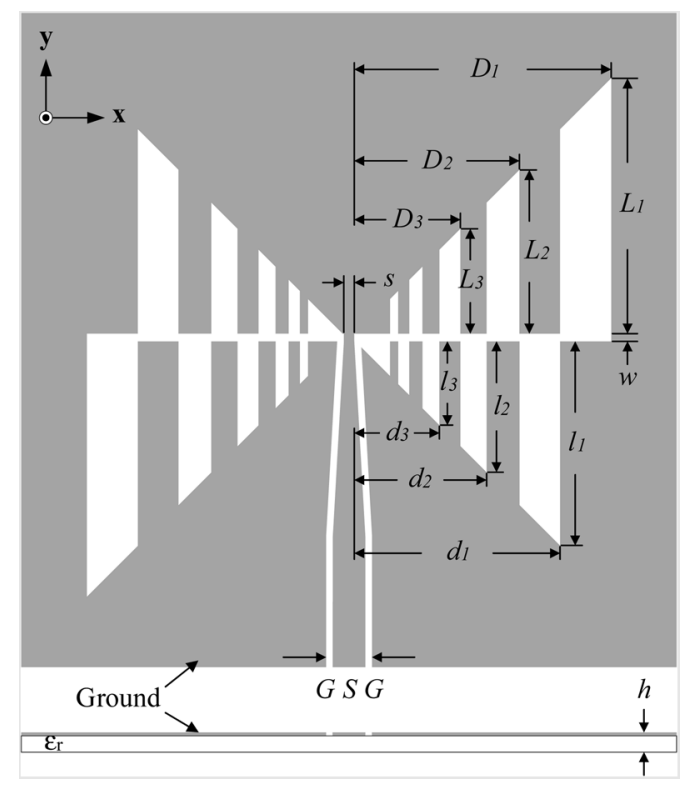

Fig. 1. Geometry of log-periodic slot antenna fed by a CPW.

\section{ANTENNA DESIGN}

The geometry of the proposed CPW-fed log-periodic slot antenna is depicted in Fig. 1. This antenna has a simple structure with only one layer of dielectric substrate and metallization. Regardless of the substrate layer and the feeding CPW, it is a complementary structure of the conventional planar log-periodic antenna [10] and is therefore wideband in essence. The successive slot lengths and distances are in the common ratio $\tau$

$$
\frac{L_{n+1}}{L_{n}}=\frac{l_{n+1}}{l_{n}}=\frac{D_{n+1}}{D_{n}}=\frac{d_{n+1}}{d_{n}}=\tau
$$

and

$$
\frac{l_{n}}{L_{n}}=\frac{d_{n}}{D_{n}}=\sqrt{\tau}
$$

which makes the spacing between any two adjacent vertical slots on the same side of the four sides of the antenna equal to the geometric mean of their widths. It is known that, in the conventional planar log-periodic antenna, the two feeding terminals are closely placed at the antenna center to ensure satisfactory performances up to a frequency at which the wavelength is comparable to the terminal spacing. Similarly, in our proposed design, the spacing between the slot terminals $s$ is properly reduced, and a linearly tapered CPW section is thus needed to connect the terminals and the CPW feedline. The upper frequency limit of the operating band $f_{U}$ can be expected when the terminal spacing $s$ is no longer negligible as compared to a 


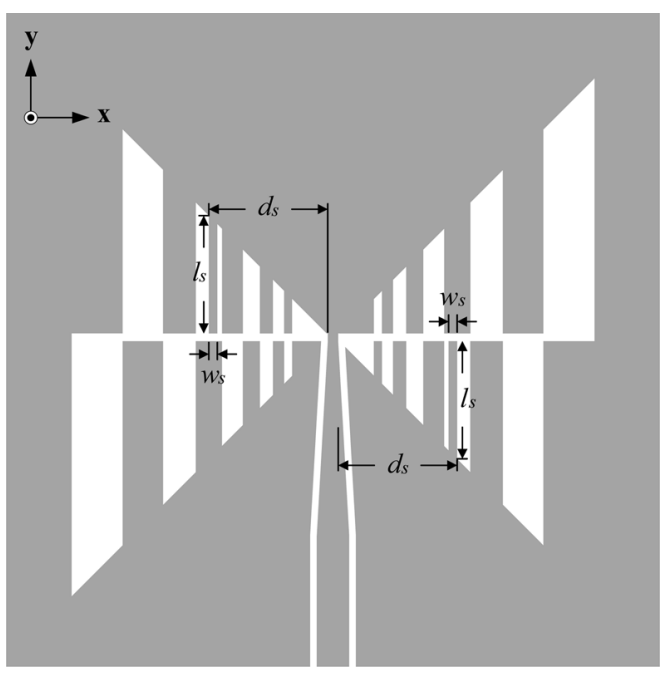

Fig. 2. Geometry of band-notched log-periodic slot antenna fed by a CPW.

guided-wavelength of the slotline which feeds the vertical slots. On the other hand, since each of the slot paths from the feeding terminals to the oblique edges of vertical slots equals uniquely a half guided-wavelength of the slotline resonating at the corresponding in-band frequency, the lower frequency limit of the operating band $f_{L}$ can be determined by the total path length from the terminal to the tip of the outermost vertical slot, namely

$$
D_{1}+L_{1}=\frac{\lambda_{g, L}}{2}
$$

where $\lambda_{g, L}$ is the guided-wavelength of the slotline at $f_{L}$. The proposed log-periodic slot antenna radiates bidirectionally in the broadside and performs stably throughout the wide bandwidth between $f_{U}$ and $f_{L}$.

In addition to the wideband feature, the CPW-fed logperiodic slot antenna can be modified to possess a narrow frequency notch within its operating band. The geometry of the band-notched design is shown in Fig. 2. Two metallic stubs of the same dimensions $l_{S}$ and $w_{S}$ are protruded from the oblique edges of the two vertical slots, which are of the same dimensions and opposite to each other both in position and direction. The protruded narrow stubs block off the vertical sections of the unwanted slot paths. This leads to a suppression of the corresponding resonant frequency. The lengths of the stubs $l_{S}$ and their distances to the feeding terminals $d_{S}$ are chosen as

$$
d_{S}+l_{S}=\frac{\lambda_{g, \text { notch }}}{2}
$$

where $\lambda_{g \text {,notch }}$ is the guided-wavelength of slotline at the center frequency of the notch. Since the insertion of the two stubs influences only the slot path directly related to the notched frequency, the resultant radiation performances as well as the impedance responses remain nearly unchanged throughout the operating band, except at the notched frequencies.

\section{EXPERIMENTAL RESULTS IN FREQUENCY DOMAIN}

Both the original and the band-notched log-periodic slot antennas fed by the CPW are designed and implemented for

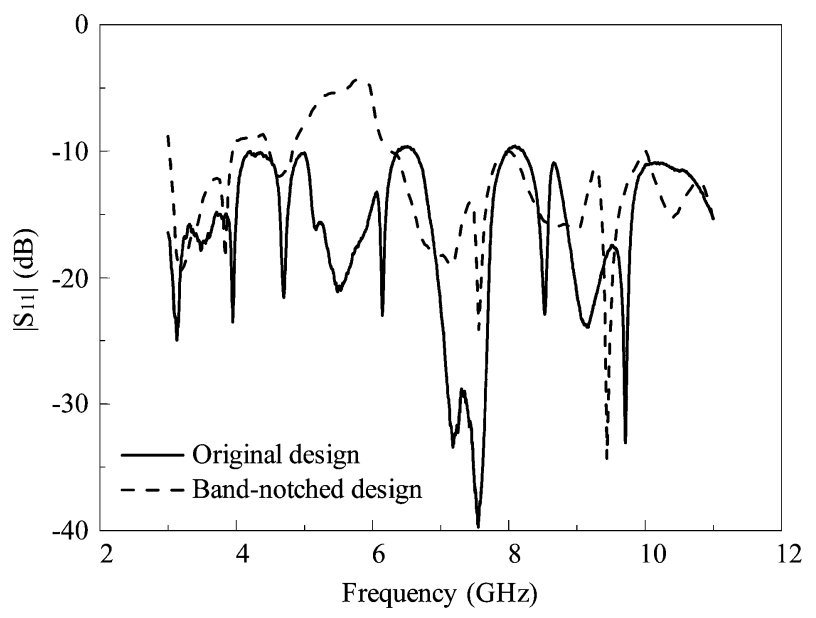

Fig. 3. Measured input return losses of the prototype antennas.

the UWB radio systems. The operating bands of both antennas should cover the 3.1 to $10.6 \mathrm{GHz}$ band, and the band-notched design should have an extra frequency notch between 5 and $6 \mathrm{GHz}$. All geometric parameters of the original design are the same as those of the band-notched one except for the protruded stubs. They are fabricated on the same RT/Duroid 5880 substrate with dielectric constant $\varepsilon_{r}=2.2$, loss tangent $\tan \delta=0.0009$, and thickness $h=1.575 \mathrm{~mm}$. In both designs, the widths of the strip and gap of the 50- $\Omega$ CPW feedline, $S$ and $G$, are chosen to be 5.0 and $0.2 \mathrm{~mm}$, respectively. To simplify the design process, $D_{n}$ is assumed to equal $L_{n}$; and consequently, $d_{n}$ equals $l_{n}$. The outer length of the outermost vertical slot $L_{1}$ is then calculated from (3), which yields $L_{1}=24.0 \mathrm{~mm}$. Also, the common ratio $\tau=0.63$, the terminal spacing $s=0.4 \mathrm{~mm}$, the widths of the horizontal thin slotlines $w=0.4 \mathrm{~mm}$, and the slot number on one of the four sides of the antenna $n=8$ for both prototype antennas. Regarding the protruded stubs in the band-notched design, the length $l_{S}$ given by (4) equals $11.1 \mathrm{~mm}$, which is about a quarter guided-wavelength of the slotline at $5.5 \mathrm{GHz}$, while the stub width $w_{S}$ is properly adjusted to be $0.6 \mathrm{~mm}$ for a notch bandwidth of approximately $1 \mathrm{GHz}$. As a consequence, the band-notched prototype antenna has a rejected frequency band from 5 to $6 \mathrm{GHz}$.

Measured input return losses of the two prototype antennas are shown and compared in Fig. 3. It is clear that the response curve of the original design stays below $-10 \mathrm{~dB}$ across the entire band of 3.1 to $10.6 \mathrm{GHz}$. The band-notched design has similar in-band responses, but within 5 to $6 \mathrm{GHz}$, there is a steep rise followed by a rapid drop above $-10 \mathrm{~dB}$ resulting in a rejected frequency band. Due to the similarity between the radiation patterns of the two antennas, only those of the original design measured at some in-band frequencies are plotted in Fig. 4. As this figure indicates, the broadside and bidirectional radiation patterns are quite stable throughout the operating band. Meanwhile, the cross-polarization levels around the broadside directions remain about $10 \mathrm{~dB}$ lower than the co-polarized components. The broadside gain variations for the two antennas throughout the entire band are $4.8 \pm 1.9 \mathrm{dBi}$, except in the notched band for the notched design. Because of these high gains, though omnior unidirectional radiation patterns may be more applicable, the bidirectional patterns of the proposed antennas are still suitable for UWB applications. 


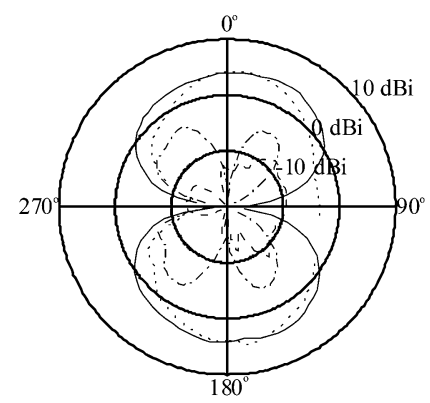

(a)

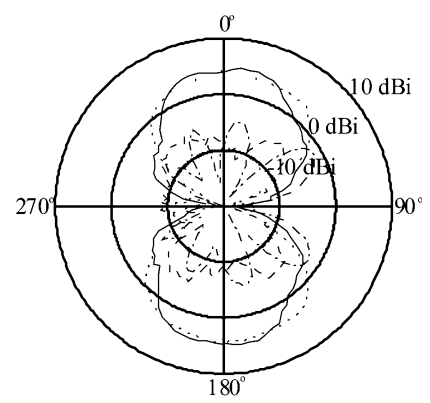

(b)

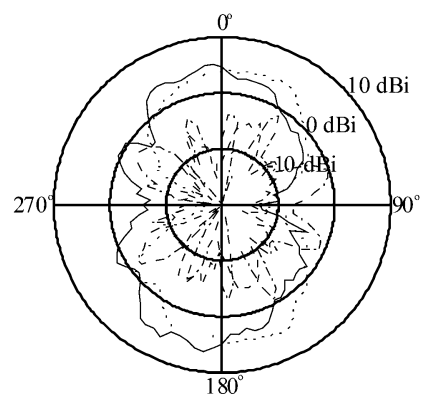

(c)

Fig. 4. Gain patterns of the prototype antenna measured at (a) 4, (b) 7, and (c) $10 \mathrm{GHz}$. Solid line: E-plane $(x-z$ plane) co-polarization. Dashed line: E-plane cross-polarization. Dotted line: H-plane ( $y$ - $z$ plane) co-polarization. Dashed-dotted line: H-plane cross-polarization.

The antenna transfer functions of both prototype antennas, which quantitatively describe how the spectrum of the incident wave will be altered by the antenna, are also investigated. In order to directly relate the antenna transfer functions to the measurable antenna parameters, a dimensionless normalized antenna transfer function $H_{\mathrm{N}, \mathrm{AUT}}(f)$ [11] in a fixed direction, say $\theta=180^{\circ}$ in this study, is defined as

$$
H_{\mathrm{N}, \mathrm{AUT}}(f)=\frac{\sqrt{4 \pi}}{\lambda} \cdot \Im\left\{h_{\mathrm{N}, \mathrm{AUT}}(t)\right\}
$$

where $h_{\mathrm{N}, \mathrm{AUT}}(t)$ is the normalized impulse response of the antenna under test (AUT) in the specified direction. Referring to [11]-[13], the normalized antenna transfer function $H_{\mathrm{N}, \mathrm{AUT}}(f)$ can be written in the forms

$$
\begin{aligned}
H_{\mathrm{N}, \mathrm{AUT}}(f) & =\left|H_{\mathrm{N}, \mathrm{AUT}}(f)\right| \cdot e^{j 2 \pi f \tau(f)} \\
& =\frac{\sqrt{4 \pi}}{\lambda} \cdot \frac{\left|H_{\mathrm{AUT}}(f)\right|}{\sqrt{f_{g}}} \cdot e^{j 2 \pi f \tau(f)} \\
& =\sqrt{G_{\mathrm{AUT}}(f)} \cdot e^{j 2 \pi f \tau(f)}
\end{aligned}
$$

where $f_{g}$ is the ratio of the characteristic impedance of the measurement system to the intrinsic impedance of free space and $\tau(f), H_{\mathrm{AUT}}(f)$, and $G_{\mathrm{AUT}}(f)$ are the group delay, effective height, and antenna gain of the AUT in the fixed direction, respectively. By carefully calibrating the measurement system and employing the gain-transfer method, both magnitudes and group delays of the normalized antenna transfer functions, $\left|H_{\mathrm{N}, \mathrm{AUT}}(f)\right|$ and $\tau(f)$, of the prototype antennas at $\theta=180^{\circ}$ are obtained and illustrated in Fig. 5. Note in Fig. 5 that $\left|H_{\mathrm{N}, \mathrm{AUT}}(f)\right|$ of the two prototype antennas remain satisfactory and stable throughout the 3.1 to $10.6 \mathrm{GHz}$ band and are nearly

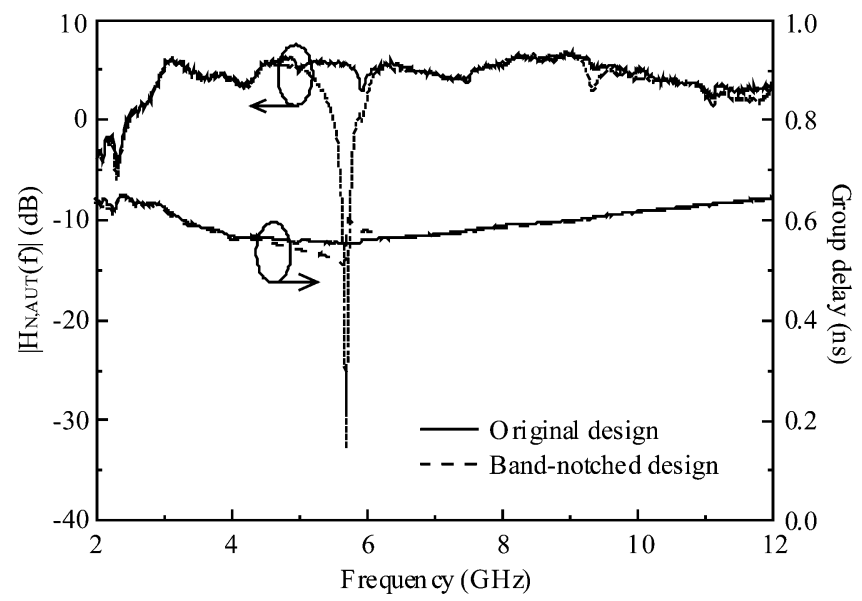

Fig. 5. Magnitudes and group delays of the normalized antenna transfer functions of the prototype antennas at $\theta=180^{\circ}$. (Step size of frequency: $12.5 \mathrm{MHz}$ ).

the same regardless of the notch centered at $5.5 \mathrm{GHz}$. Due to the dispersive essence of the log-periodic structures [8], [9], the group delays $\tau(f)$ shown in Fig. 5 are not invariable. However, fortunately, the variations in group delays of the proposed antennas are reasonably suppressed. This may be explained by the compact antenna size or the short slot path from the central feed point to the inner edge of the farmost vertical slot element, which is only $15.12 \mathrm{~mm}\left(d_{1}\right)$ in our prototype antennas. As Fig. 5 illustrates, the original design exhibits a flat response section between 4.5 and $6.5 \mathrm{GHz}$ with a minimum group delay of $0.554 \mathrm{~ns}$ and a linearly and steadily increasing section beyond that. The band-notched design possesses similar increasing responses beyond $6.5 \mathrm{GHz}$ and a sharp transition in the notched band, which significantly lowers the minimum group delay to be $0.512 \mathrm{~ns}$. Consequently, in the observed frequency band of 2 to $12 \mathrm{GHz}$, the varying range of the group delays for the band-notched design is greater than that for the original design, and they are 0.140 and $0.091 \mathrm{~ns}$, respectively.

\section{TIME-DOMAIN CHARACTERISTICS}

Since, for the pulsed UWB radios, the time-domain response of the antenna is vital to the system performances, the present section focuses on the time-domain characteristics of the proposed antennas. The simple procedures for obtaining the timedomain responses are as follows: the spectrum of an incident pulse is multiplied by the normalized antenna transfer functions $H_{\mathrm{N}, \mathrm{AUT}}(f)$ of the prototype antennas acquired above and then converted to time domain. To comply with the required FCC indoor emission mask, the waveform of the incident pulse arriving at the receiving antenna $s_{i}(t)$ is determined to be the fourth derivative of a Gaussian function [11]

$s_{i}(t)=A\left[3-6\left(\frac{4 \pi}{T^{2}}\right) t^{2}+\left(\frac{4 \pi}{T^{2}}\right)^{2} t^{4}\right] \cdot e^{-2 \pi(t / T)^{2}}\left(\frac{\mathrm{V}}{\mathrm{m}}\right)$

where $A=0.1$ and $T=0.175$ ns. Fig. 6(a) illustrates the incident waveform $s_{i}(t)$. With the use of $(7)$ and $H_{\mathrm{N}, \mathrm{AUT}}(f)$ 


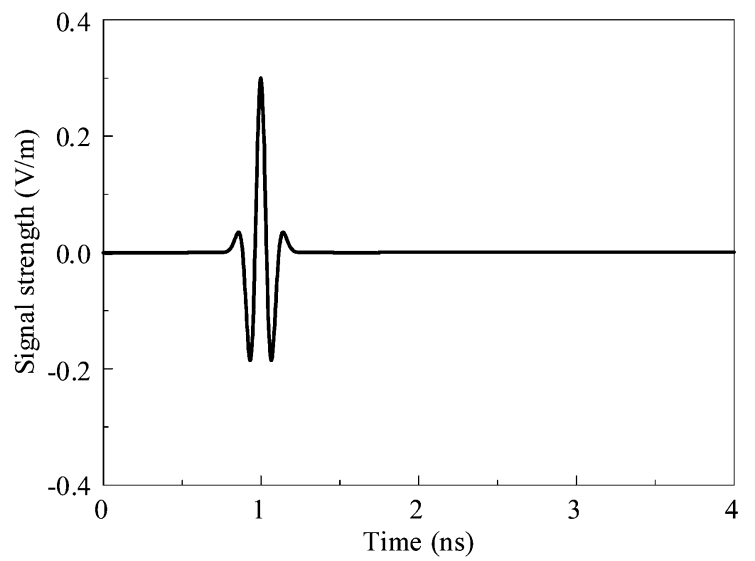

(a)

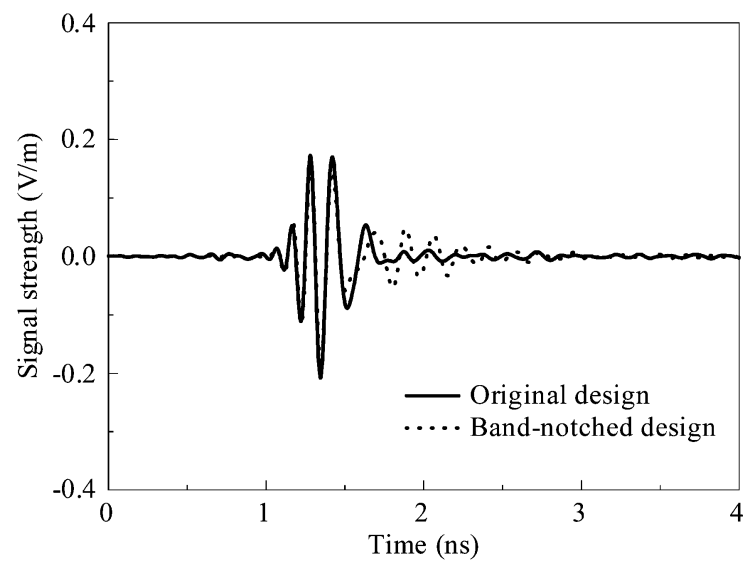

(b)

Fig. 6. (a) Waveform of the incident pulse arriving at the receiving antenna from $\theta=180^{\circ}$. (b) Received waveforms at the antenna terminals of the prototype antennas.

obtained in Section III, the output waveform at the receiving antenna terminal $s_{o}(t)$ can thus be evaluated through

$$
s_{o, \mathrm{AUT}}(t)=\Im^{-1}\left\{\Im\left\{s_{i}(t)\right\} \cdot H_{\mathrm{N}, \mathrm{AUT}}(f)\right\}\left(\frac{\mathrm{V}}{\mathrm{m}}\right) .
$$

The output waveforms of the original and the band-notched designs are depicted and compared in Fig. 6(b). The received pulses of the two designs, though slightly distorted, remain acceptable. The main difference between them is that the band-notched design exhibits a stronger late-time oscillation or ringing, which may be attributed to the greater varying range of the group delays.

\section{CONCLUSION}

The CPW-fed log-periodic slot antennas with and without a notched frequency band have been proposed and investigated in this paper. The design with a notch was obtained by simply inserting two additional stubs into the one without. The main distinction between them is that the impedance and radiation performances of the band-notched antenna degrade rapidly within the notch. Except that, the in-band operation of the two antennas are very similar, including the wideband input match, the dumbbell-shaped and stable radiation patterns, and the satisfactory and flat gain responses. In time domain, the well-behaved responses manifest the applicability of the proposed antennas in the pulsed systems. These characteristics and the simple, compact, and uniplanar configuration make the proposed log-periodic slot antennas highly suitable for use in the UWB radio systems.

\section{REFERENCES}

[1] FCC, "FCC first report and order on ultra-wideband technology,", 2002.

[2] K. Siwiak, "Ultra-wide band radio: Introducing a new technology," in Proc. IEEE Vehicular Technology Conf., vol. 2, May 2001, pp. 1088-1093.

[3] D. Porcino and W. Hirt, "Ultra-wideband radio technology: Potential and challenges ahead," IEEE Commun. Mag., vol. 41, no. 7, pp. 66-74, Jul. 2003.

[4] Y. Kim and D.-H. Kwon, "CPW-fed planar ultra wideband antenna having a frequency band notch function," Electron. Lett., vol. 40, no. 7, pp. 403-405, Apr. 2004.

[5] W.-J. Lui, C.-H. Cheng, Y. Cheng, and H. Zhu, "Frequency notched ultra-wideband microstrip slot antenna with fractal tuning stub," Electron. Lett., vol. 41, no. 6, pp. 294-296, Mar. 2005.

[6] K.-H. Kim, Y.-J. Cho, S.-H. Huang, and S.-O. Park, "Band-notched UWB monopole antenna with two parasitic patches," Electron. Lett., vol. 41, no. 14, pp. 783-785, Jul. 2005.

[7] W.-S. Lee, W.-G. Lim, and J.-W. Yu, "Multiple band-notched planar monopole antenna for multiband wireless systems," IEEE Microw. Wireless Components Lett., vol. 15, no. 9, pp. 576-578, Sep. 2005.

[8] W. Sorgel, C. Waldschmidt, and W. Wiesbeck, "Transient responses of a Vivaldi antenna and a logarithmic periodic dipole array for ultra wideband communication," in IEEE AP-S Int. Symp. Dig., vol. 3, Jun. 2003 , pp. $592-595$

[9] S. Licul, J. Noronha, W. Davis, D. Sweeney, C. Anderson, and T. Bielawa, "A parametric study of time-domain characteristics of possible UWB antenna architectures," in Proc. IEEE Vehicular Technol. Conf., vol. 5, Oct. 2003, pp. 3110-3114.

[10] R. S. Elliott, Antenna Theory and Design, Revised ed. Hoboken, NJ: Wiley-Interscience, 2003, pp. 379-385.

[11] T.-G. Ma and S.-K. Jeng, "Planar miniature tapered-slot-fed annular slot antennas for ultrawide-band radios," IEEE Trans. Antennas Propagat., vol. 53, no. 3, pp. 1194-1202, Mar. 2005.

[12] B. Scheers, M. Acheroy, and A. V. Vorst, "Time-domain simulation and characterization of TEM horns using a normalized impulse response," Proc. Inst. Elect. Eng.-Microw. Antennas Propagat., vol. 147, no. 6, pp. 463-468, Dec. 2000.

[13] C. Baum, E. Farr, and C. Frost, "Transient gain of antennas related to the traditional continuous-wave (CW) definition of gain," Proc. UltraWideband Short-Pulse Electromagnetics 4, pp. 109-118, Jun. 1998. 